\title{
Evaluation of Protein Adsorption on Chitosan Surfaces with Reflectometry Interference Spectroscopy
}

\author{
Xiao Ying Lü*, Yan Huang and Chao Qun Ma
}

Key Laboratory of Molecular and Biomolecular Electronics of the Ministry of Education, P. R. China. Southeast University, 210096, Nanjing, China. Tel: (86)-25-3793430. Fax: (86)-25-7712719.

E-mail: luxy@seu.edu.cn. Homepage: http://www.lmbe.seu.edu.cn

* Author to whom correspondence should be addressed.

Received: 4 September 2001 / Accepted: 25 September 2001 / Revised: 10 October 2001 /

Published: 12 October 2001

\begin{abstract}
Using a biomedical sensor setup RIfS we have investigated the kinetic behavior of human albumin (Alb), human fibrinogen (Fib), and human immunoglobulin G (IgG) adsorbed onto surfaces of chitosan. Polystyrene (PS) was used as the control material in this study. The optical thickness of three kinds of proteins measured by RIfS was related to their molecular dimensions and potential orientations on a film surface. According to the operation principle of RIfS and the molecular dimensions of three kinds of proteins, the adsorbed layers of proteins onto the surface of chitosan and PS was calculated by using a newly introduced equation. The microstructure of the chitosan and polystyrene film and the surfaces with adsorbed proteins were imaged by atomic force microscopy (AFM). With AFM analyses the lateral distribution of the protein molecules on surfaces have been recognized. The results show that the number of adsorbed layers of the three proteins on the surface of chitosan are 0.635 for Alb, 0.158 for Fib and 0.0967 for IgG, and of polystyrene are: 0.577 for $\mathrm{IgG}, 0.399$ for Fib, 0.336 for Alb. This study confirmed that RIfS is a useful tool for the analysis of plasma proteins adsorbed on a surface of biomaterials. Results show that at first on the surface of chitosan film much more Alb than Fib was adsorbed which demonstrated that chitosan has a antithrombus function. Secondly, on the surface of chitosan film more $\mathrm{Alb}$ and less Fib were adsorbed than on the surface of PS film, which demonstrated that chitosan has a better blood compatibility than polystyrene. Thirdly, the calculated layer number of the three proteins indicated that on both chitosan and PS substrates monolayer coatings form.
\end{abstract}

Key words: Biomedical sensor, RIfS, chitosan, Protein adsorption, Biomaterials 


\section{Introduction}

Optical interferomertry is well known for the measurement of physical parameters such as speed and distance. Further, light interference was used for visual detection of antigen-antibody reactions [1]. Spectral interferometry has been considered as an interesting approach to detecting sensitively and rapidly any changes of optical path length in thin polymer films caused by antigen-antibody interaction in immunochemical sensing [2]. After the development of surface plasmon resonance (SPR) $[3,4]$, another successful optical approach, Reflectometry Interference Spectroscopy (RIfS), was introduced as a highly sensitive and robust technique for direct label-free monitoring of the interaction of biomolecules [5,6,7].

RIfS is based on the spectral distribution of white-light reflectance from transparent thin layers. A distinct reflectance pattern with alternating maxima and minima results from the interference of beams partially reflected from each interface of the interference layer. Binding detection with RIfS shows little temperature dependence, allows avoidance of gold layers, and is promising for high throughput screening. The simple test format and high sensitivity makes RIfS attractive for detecting and characterizing the interaction of biomolecules. Biological material deposited at the surface during a binding event increases the optical thickness of the interference layer, leading to a shift in the interference pattern. This approach allows on-line monitoring of binding reactions with high resolution [8]. The main advantages of the RIfS transducer are its ruggedness, the small active area, and the simple construction [9].

A sensitive dynamic determination made on gaseous or liquid hydrocarbons as well as immunoreactions with RIfS was reported in 1993 [6]. Then, an investigation of the specific binding of low molecular weight ligands to immobilized receptors was carried out by using RIfS; two model systems were studied in which the binding event was successfully detected [10]. Next, RIfS was utilized for studying the hybridization of antisense with its complementary target sequences and especially to investigate the interaction of terminal- and backbone-modified antisense ONs targeted to multidrug resistance1b-mRNA [8]. Other applications with RIfS were in a dyeless optical detection of ammonia in the gas phase, by using pH-responsive polymers. Also, clinical detection of HbsAGb was accomplished [11, 12]. Recently, the application of RIfS for the evaluation of the biocompatibility of biomaterials has been described [13].

Since the protein adsorption on the surface of biomaterials is an important factor for the adhesion of platelet and biocompatibility, the characterization of adsorbed proteins on the surface of various biomaterials, such as biopolymers, alloys, ceramics etc., has been extensively investigated by means of different techniques [14-26].

Chitosan is a natural source polymer prepared by N-deacetylation of chitin, which is the main structural component of crab and shrimp shells [27]. Intensive investigations have been carried out for the structure, the chemistry, the biological properties, and the applications of chitosan [28].

Chitosan has been widely used as a new biomaterial since it is safe and has a good biocompatibility with the human body [29-35]. Chitosan also has a haemostatic function [36] and may have an antithrombogenic function. Some investigators reported the platelet adhesion on the surface of modified chitosan $[37,38]$. However, the protein adsorption occurrs before the platelet adhesion and is the first step of thrombosis. The aim of this study is to evaluate the protein adsorption behavior of 
human albumin (Alb), human fibrinogen (FIb), and human IgG on chitosan, by using RIfS. The kinetics of the adsorption process is investigated. Polystyrene is used as the control material, since its surface chemistry is well defined and it is frequently used as a model system in protein adsorption studies [39].

\section{Materials and Methods}

\subsection{RIfS Apparatus}

The RIfS setup used has been developed in our laboratory [40]. The main elements of the setup were a CCD spectrometer with a bifurcated optical fiber and an adsorption cell with a volume of about $30 \mu \mathrm{L}$. The bifurcated optical fiber was used to illuminate the transducer chip and to collect the light reflected from the chip. The chip is a glass substrate on which a $20-\mathrm{nm} \mathrm{Ta}_{2} \mathrm{O}_{5}$ film was deposited in order to enhance the interference efficiency. An original software was used to control the collection of full spectra, to analyze the experimental data in real-time, and to draw the experimental curves on-line.

\subsection{Preparation of Chitosan and PS films}

Chitosan with a degree of deacetylation of $93.2 \%$ and of an average molecular weight of 750,000 Da was purchased from Ocean Institute of Jiangsu, China. The material is powder form with 80-100nm granularities and has a white color. The thin films of chitosan exposed to proteins were prepared by the spin-coating method. At first, the chitosan was immersed in $0.5 \%$ acetic acid (Factory of Chemical Reagents of Nanjing, China) with $10 \mathrm{mg} / \mathrm{mL}$. After the chitosan was dissolved completely, the solution was filtered with a middle speed qualitative filter paper (Factory of Fuyang Special Paper of Hangzhou, China). Then, $50-\mu \mathrm{L}$ chitosan solution was pipetted out and dropped onto the surface of the transducer chip $(1.2 \mathrm{~cm} \star 1.2 \mathrm{~cm})$. A chitosan film was spin-coated on the transducer chip using a spinning-machine at $1700 \mathrm{rpm}$. Typically, the film thickness was $\sim 650 \mathrm{~nm}$.

Polystyrene (Nunc Inc. Denmark) was dissolved in toluene ( Factory of Chemical Reagents of Nanjing, China) at a concentration of $40 \mathrm{mg} / \mathrm{mL}$. The coating process of polystyrene film on the transducer chip is same with the chitosan film. All materials and reagents as well as chemicals were of analytical grade or better.

\subsection{Proteins}

Three kinds of proteins were used : human albumin (Alb), human fibrinogen (Fib), and human IgG (Sigma, Dorset, UK). For each measurement a protein solution was prepared freshly in $10 \mathrm{mM}$ phosphate buffer solution (PBS, $\mathrm{pH}=7.4$ ) of a concentration of $0.1 \mathrm{mg} / \mathrm{mL}$. Deionized water was used to prepare the aqueous solutions.

\subsection{Protein Adsorption Measurement}

The protein adsorption measurement has been performed as follows: the sensor chip coated with chitosan/PS film was mounted into the adsorption cell carefully and fixed with two clips. Then, the 
peristaltic pump was started to drive PBS $(\mathrm{pH}=7.4)$ flow into the adsorption cell. The PBS cleaned the surface of the chitosan/PS film, drove out air bubbles that may be present in the adsorption cell. Most importantly, it produced a physiological $\mathrm{pH}$-environment for the adsorption of proteins. After this treatment, the protein solution flowed into the adsorption cell at a rate of $200 \mu \mathrm{L} / \mathrm{min}$ and the kinetic adsorption process of proteins on the surface of the chitosan/PS film can be oberseved in situ on the monitor of the computer. Until the kinitic adsorption curve of proteins became stable, PBS was pumped into the adsorption cell again to wash the proteins which did not adsorb tightly onto the material surface. The wash handing would get a response on the adsorption curve. The mark on the $\mathrm{Y}$ axis of the Figs.1-4 give the thickness of the proteins adsorbted on the chitosan/PS film. For each kind of proteins, at least three tests were performed $(n=3)$.

\subsection{Calculation of the Adsorbed Layer of Protein}

Since the molecular dimensions of various proteins are different, a normalized treatment of the experimental data was performed using a previously defined equation [13] in order to obtain the effective adsorbed layer number $L_{\mathrm{p}}$ (Eq.1):

$$
L_{\mathrm{p}}=\frac{\overline{x_{\mathrm{p}}}}{D_{\mathrm{p}}}
$$

where $D_{\mathrm{p}}$ is the molecular dimension of a single protein and $\overline{x_{\mathrm{p}}}$, the average thickness of each type of proteins on the surface of the material intended to be probed with RIfS.

\subsection{AFM Imaging}

The microstructure of the chitosan and polystyrene films and the surfaces with adsorbed proteins were imaged with a commercial atomic force microscope (AFM, Nanoscope IIIa, Digital Instrument, Santababara, USA). The instrument was operated in Tapping Mode using silicon cantilevers oscillating with the average amplitude of $100 \mathrm{~nm}$ and a resonance frequency between 200 and $450 \mathrm{kHz}$. The scanning rate selected was $1 \mathrm{~Hz}$. All images presented in this paper were obtained repeatedly and were stable under the experimental conditions.

\section{Results and Discussion}

\subsection{Kinetics of Protein Adsorption onto PS Films}

In Fig. 1 the kinetic adsorption curves of human Alb, IgG and Fib onto the surface of a PS film are shown. The curves evidence that the adsorption behavior of the three proteins onto PS film are similar: initially, the processes of all three proteins increase quickly in the initial stage and reach their equilibrium values after about 500 s. Secondly, after washed with PBS the adsorption curves have different tendencies: the curve of $\mathrm{IgG}$ falls about $0.2 \mathrm{~nm}$, which means some loosely adsorbed molecules were removed from the PS film. Nevertheless, the curves of Alb and Fib show almost no 
response, which shows the adsorption process of albumin and fibrinogen onto the surface of PS film have not been affected.

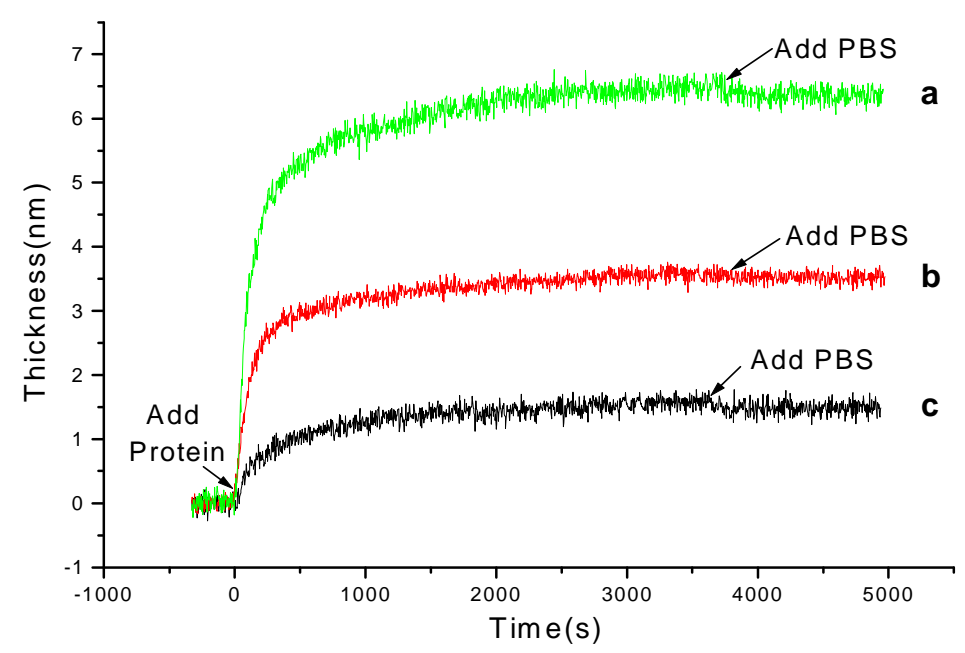

Figure 1. Kinetic curves of protein adsorption onto the surfaces of PS film: a) IgG, b) Fib, c) Alb.

This result can be derived from the difference of sizes and structures of three kinds of proteins. The adsorbed process of proteins onto the surface of biomaterials is pictured schematically in Fig. 2. According to Green et al. [39], albumin is a globular protein and the potential orientation on a surface is ca. $4 \mathrm{~nm}$. Fibrinogen has a rod-like structure with approximately 10-nm width and the molecules may lie planar on the biomaterials interface. IgG has a relatively globular Y shaped structure with a height of $\sim 12 \mathrm{~nm}$. Therefore, the different molecular dimensions of the three proteins are responsible for the difference of the thickness. The smallest height of Alb molecules, for example, yields the thinnest film. The intermediate molecular dimension of Fib results in an intermediate optical thickness between Alb and IgG. The random orientation of IgG molecules adsorbed on a PS film results in a thickest adsorptive layer as measured by RIfS. The curve family is similar to that reported by Green et al. for PS material by an analysis using SPR [39] and by us for hydroxyapatite using RIfS.

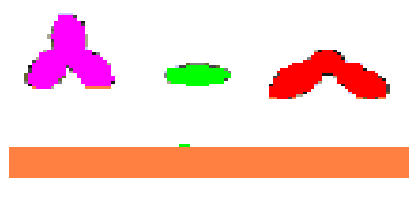

(a)

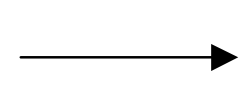

(b)

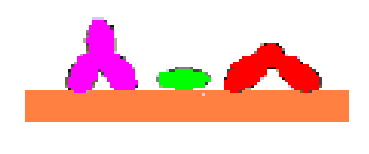

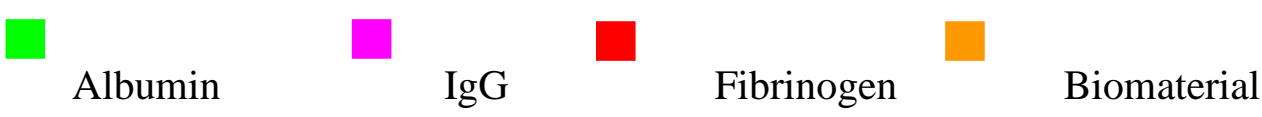

Figure 2. Sketch map of adsorbed process of proteins onto the surface of biomaterials:

(a) before and (b) after adsorption. 


\subsection{Kinetics of Protein Adsorption onto Chitosan Films}

The kinetic adsorption curves of human Alb, Fib, and IgG on the surface of a chitosan film are shown in Fig. 3-5. According to these curves the adsorption behavior of three kinds of proteins onto chitosan is dissimilar. For Alb, the adsorption processes amplifies slowly in the initial stage and reaches its stable values after about $8000 \mathrm{~s}$. This means that during this period the adsorption of albumin on chitosan continued. Nevertheless, for Fib and IgG the adsorption processes is fast in the initial stage and reaches its stable values after about $1500 \mathrm{~s}$, which means that the adsorption process of Fib and IgG on chitosan films reaches saturation fast. After washed with PBS, the curves of Alb, Fib, and $\mathrm{IgG}$ fall apparently, which indicates more loosely adsorbed molecules were removed from the chitosan film.

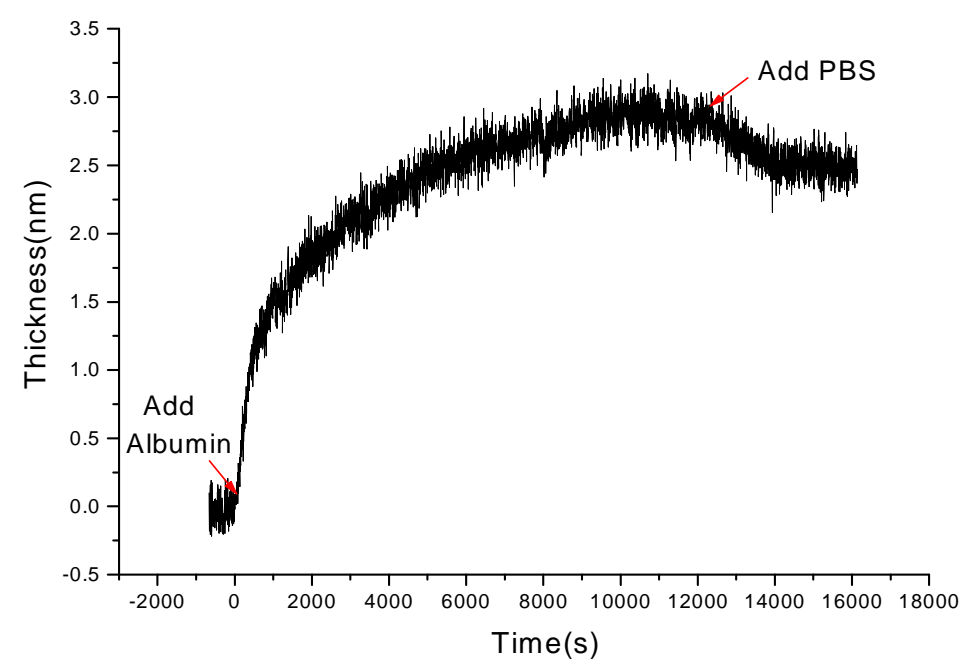

Figure 3. Kinetic curves of adsorbed Alb on the surface of chitosan film.

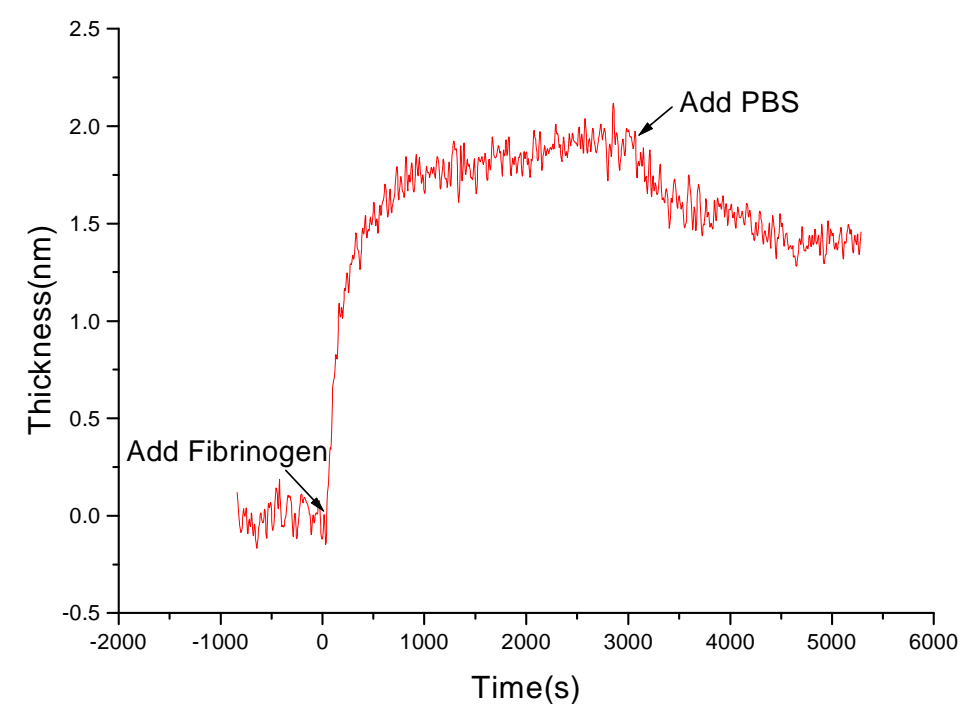

Figure 4. Kinetic curves of adsorbed Fib on the surface of chitosan film. 


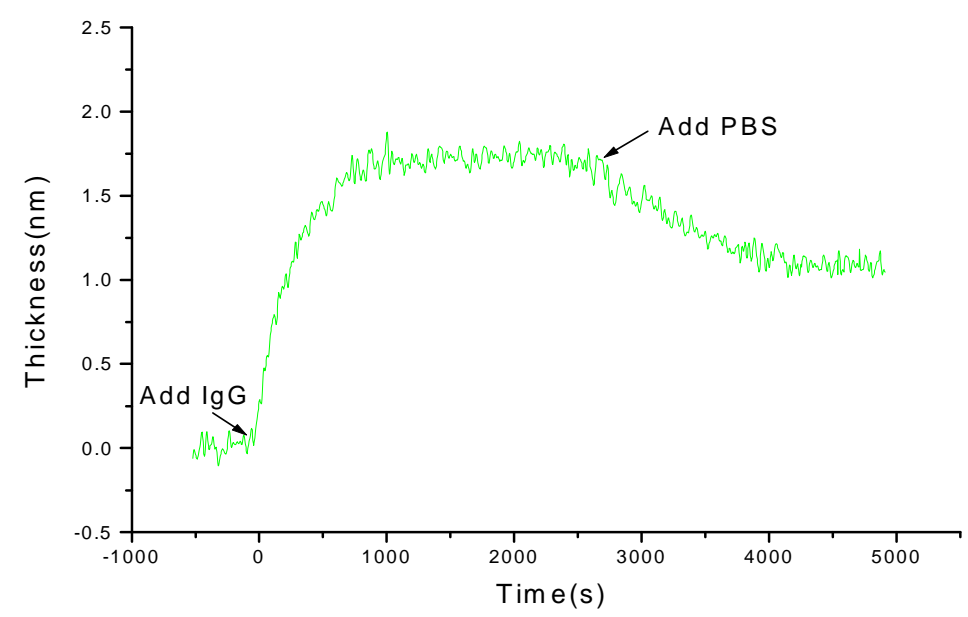

Figure 5. Kinetic curves of adsorbed IgG on the surfaces of chitosan film.

\subsection{Comparing of the Kinetic Curves on Chitosan and PS}

Fig. 6-8 compare the adsorption of the three kinds of proteins onto the surface of chitosan and PS films. The curves reveal that on the surface of chitosan film more Alb was adsorbed than on the surface of PS film, and conversely, on the surface of PS film more Fib and IgG were adsorbed than on the surface of chitosan films.

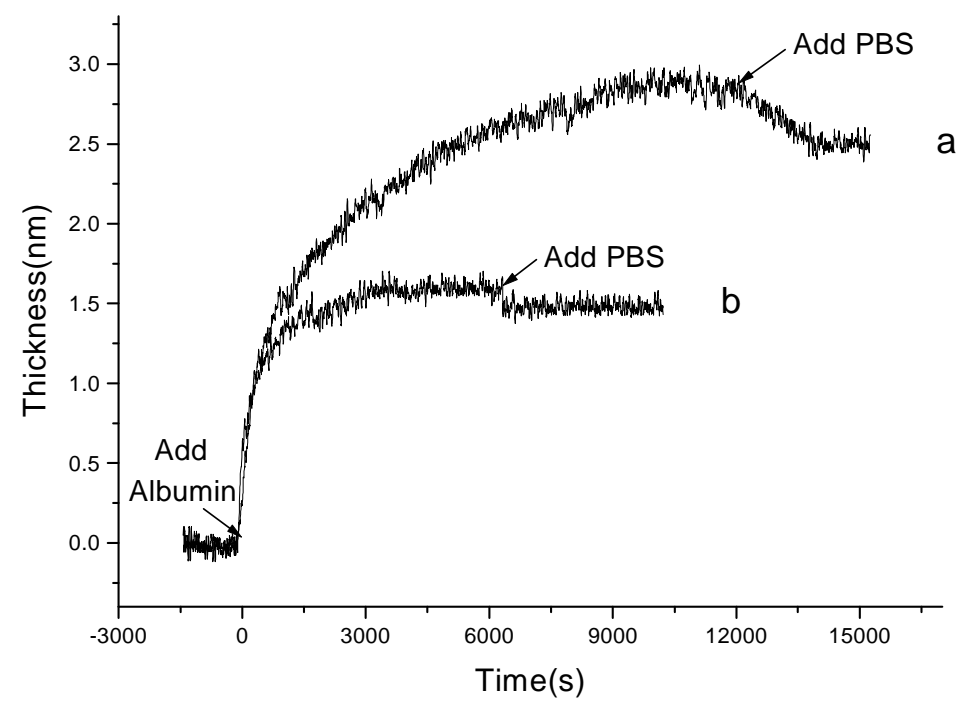

Figure 6. Adsorbed Alb on the surfaces of chitosan and PS: a) chitosan, b) PS. 


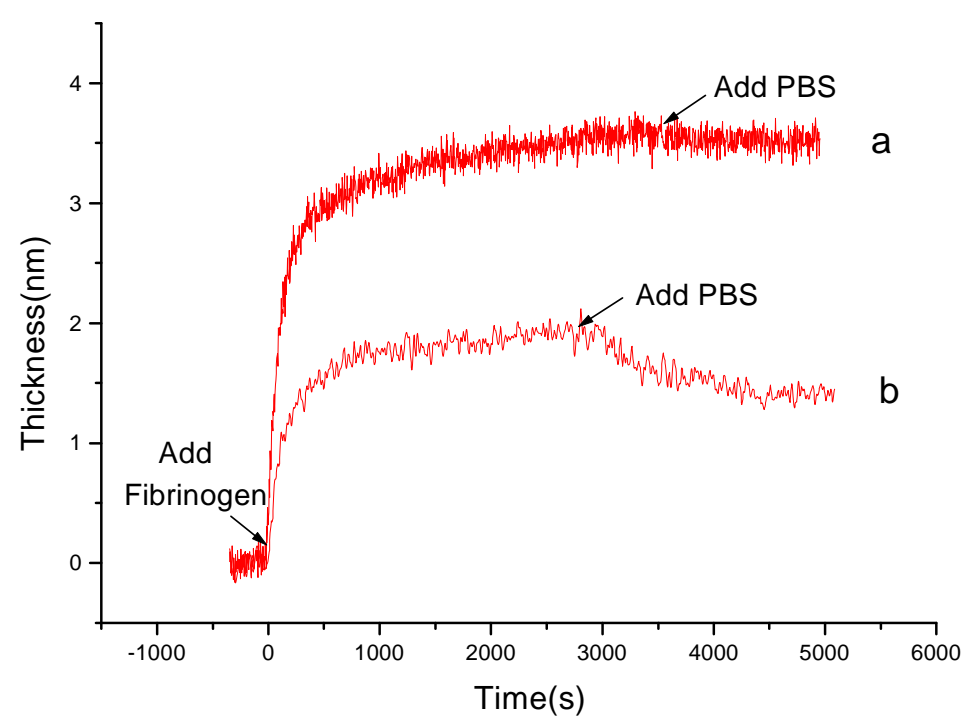

Figure 7. Adsorbed Fib on the surfaces of chitosan and PS: a) PS, b) chitosan.

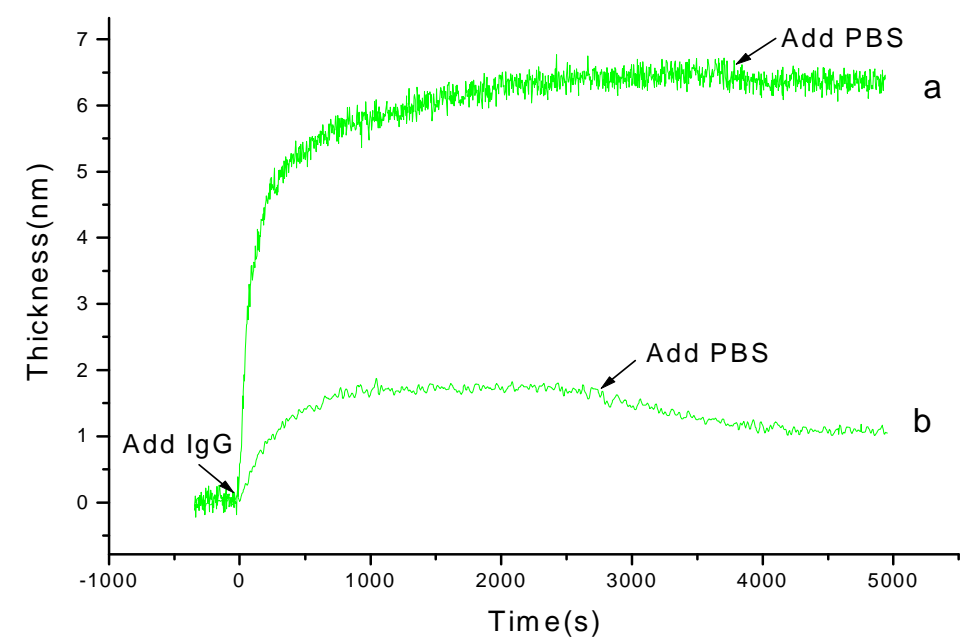

Figure 8. Adsorbed IgG on the surfaces of chitosan and PS: a) PS, b) chitosan.

\subsection{Adsorbed Layers of Alb, Fib, and IgG on Chitosan and PS Films}

As discussed above, each adsorption curve indicates the thickness of one particular protein adsorbed onto the surface of one kind of material. Thus, the adsorptive thickness of one kind of proteins on films of different materials can be compared directly according to the curve family as in Figs. 6-8. But with the RIfS thickness a comparison of the adsorbed amount of different proteins on the same materials cannot be carried out directly. Since the structure and size of three proteins are very different. After a normalized treatment of the experimental data using the equation, the thickness of the adsorbed protein layers was calculated, which indicates the effectively adsorbed amount of different proteins on one particular material. 
RIfS thickness of three kinds of proteins on the PS surface decreases in the order (Tab.1): IgG $(6.35 \mathrm{~nm})>\mathrm{Fib}(3.59 \mathrm{~nm})>$ Alb $(1.34 \mathrm{~nm})$. The adsorbed layer number, calculated using the equation, PS has same series: $\operatorname{IgG}(0.577)>$ Fib (0.399) > Alb. (0.336).

The thickness determined by RIfS for the three kinds of proteins on the chitosan surface decreases in the order (Tab.2): Alb (2.54nm) > Fib (1.42nm) > IgG (1.06nm). By this order a special phenomena for albumin adsorbed onto chitosan film is recognized. Despite the smallest structure the adsorbed RIfS thickness of Alb is greatest among the three kinds of proteins. This result is very different from the results of PS and of another studies [13, 39]. It indicates that chitosan adsorbed much more Alb than Fib and IgG. In this case the adsorbed layer number of three kinds of proteins on chitosan decreases in the same order: Alb (0.635) > Fib (0.158) > IgG (0.0967).

The calculated layer numbers of three kinds of proteins show that the total amount of each kind of protein adsorbed on chitosan and PS occurs as monolayer adsorption.

According to previous studies, albumin has a thrombus-resistant ability, whereas fibrinogen promotes platelet adhesion on a polymer surface [41]. The calculated number of adsorbed layers of the three proteins on chitosan and PS evidences that the chitosan material shows a higher affinity to Alb and lower affinity to Fib than PS. This means that chitosan has a better antithrombus function relative to PS films.

Table 1. RIfS thickness and adsorbed layer number of three kinds of proteins on the PS film.

\begin{tabular}{|c|c|c|c|}
\hline Thickness Proteins & $\bar{x}(\mathbf{n = 3})$ & SD & $\begin{array}{c}\text { Adsorbed } \\
\text { Layer }\end{array}$ \\
\hline Alb & 1.34 & 0.0868 & 0.336 \\
\hline Fib & 3.59 & 0.0586 & 0.399 \\
\hline $\mathrm{IgG}$ & 6.35 & 0.0430 & 0.577 \\
\hline
\end{tabular}

Table 2. RIfS thickness and adsorbed layer number of three kinds of proteins on the chitosan film.

\begin{tabular}{|c|c|c|c|}
\hline Thickness Proteins & $\bar{x}(\mathbf{n = 3})$ & SD & $\begin{array}{c}\text { Adsorbed } \\
\text { Layer }\end{array}$ \\
\hline Alb & 2.54 & 0.0193 & 0.635 \\
\hline Fib & 1.42 & 0.0220 & 0.158 \\
\hline IgG. & 1.06 & 0.0505 & 0.0967 \\
\hline
\end{tabular}

\subsection{AFM characteristic}

AFM analysis is useful for probing the lateral distribution of the protein molecules with the monolayer and confirmed indirectly the presence of the passively adsorbed protein on the surfaces of biomaterials. The AFM image in Figs. 9(a) and figure10(a) are the microstructure of the chitosan and PS surface, while in Figs. 9(b) and 10(b) the same surfaces with adsorbed protein albumin.

From the AFM image in Fig. 9(a), the pattern in the surface of chitosan material can be revealed. The AFM images of albumin coated chitosan surface in figure9(b) reveal that the most area of the surface of chitosan film was covered with the proteins. 
The AFM image of PS film in Fig. 10(a) termed as ultra-flat film [42]. The AFM image in Fig. 10(b) reveal that there is fewer albumin on PS film than on chitosan film in figure 9(b).

AFM analyses are consistent with the calculated adsorbed layer in Tab. 1. and Tab. 2.

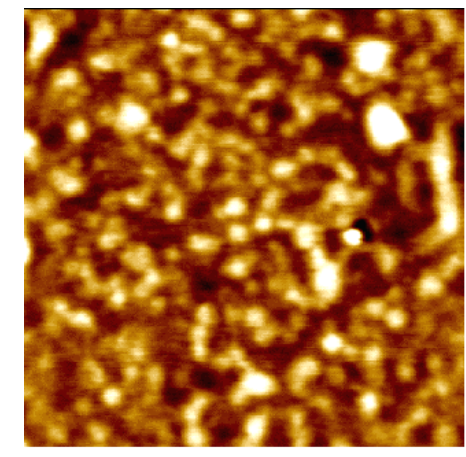

(a)

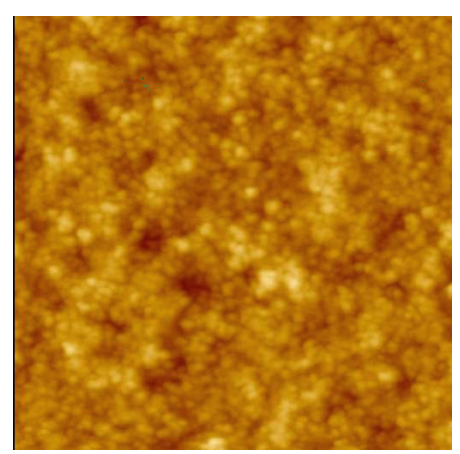

(b)

Figure 9. AFM image of microstructure of a chitosan surface (a) and with adsorbed albumin (b).

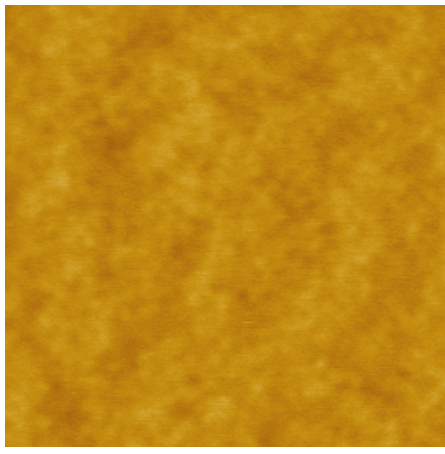

(a)

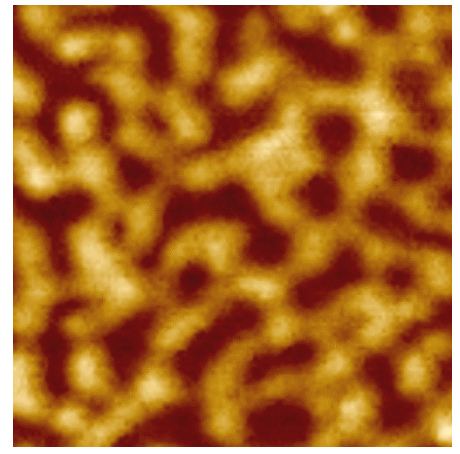

(b)

Figure 10. AFM image of microstructure of a PS surface (a) and with adsorbed albumin (b).

\section{Conclusions}

This study confirmed that RIfS is a useful tool for analysis of plasma proteins adsorbed on a surface of biomaterials. The number of adsorbed layers of three kinds of proteins on the surface of chitosan has a series: Alb > Fib > IgG and PS has a series: $\mathrm{IgG}>$ Fib > Alb. Results reported in this paper show that at first on the surface of chitosan film more Alb and fewer Fib adsorbs, which demonstrates that chitosan has a antithrombus function and secondly, on the surface of chitosan film more Alb and less Fib than on the surface of PS film, which demonstrated that chitosan has a better blood compatibility than polystyrene. And thirdly, according to the calculated layer number of the three proteins all the proteins on chitosan and on PS materials represents a monolayer adsorption.

\section{Acknowledgements}

We are thankful to the Ministry of Education of China for the support by the "Promotion Foundation for Scholars from Abroad" (1998-2000). 


\section{References}

1. Kawaguchi, T.; Shiro, T.; Iwata, K. A highly sensitive device for visual detection of antigens and antibodies by means of light interference. Sensors and Actuators B 1991, 3, 113-121.

2. Brecht, A.; Gauglitz, G.; Nahm, W. Interferometric measurement used in chemical and biochemical sensors. Analysis 1992, 20, 135-140.

3. Myszka, D.G. Kinetic analysis of macromolecular interactions using surface plasmon resonance biosensors. Current Opinion in Biotechnology 1997, 8, 50-57.

4. Green, R.J.; Frazier, R.A.; Shakesheff, K.M.; Davies, M.C.; Roberts, C.J.; Tendler, S.J.B. Surface plasmon resonance analysis of dynamic biological interactions with biomaterials. Biomaterials 2000, 21, 1823-1835.

5. Brecht, A.; Ingenhoff, J.; Gauglitz, G. Direct monitoring of antigen-antibody interactions by spectral interferometry. Sensors and Actuators B 1992, 6, 96-100.

6. Gauglitz, G.; Brecht, A.; Kraus, G.; Nahm, W. Chemical and biochemical sensors based on interferometry at thin (multi-) layers. Sensors and Actuators B 1993, 11, 21-27.

7. Schmitt, H.M.; Brecht, A.; Piehler, J.; Gauglitz, G. An integrated system for optical biomolecular interaction analysis. Biosensors \& Bioelectronics 1997, 12(8), 809-816.

8. Sauer, M.; Brecht, A.; Charissé, K.; Maier, M.; Gerster, M.; Stemmler, I.; Gauglitz, G.; Bayer, E. Interaction of Chemically Modified antisense oligonucleotides with sense DNA: a label-free interaction study with reflectometric interference spectroscopy. Analytical Chemistry 1999, 71, 2850-2857.

9. Brecht, A.; Gauglitz, G. Optimised layer systems for immunosensors used on the RIFS transducer. J Anal Chem. 1994, 349, 360-366.

10. Piehler, J.; Brecht, A.; Gauglitz, G.; Maul, C.; Grabley, S.; Zerlin, M. Specific binding of low molecular weight ligands with direct optical detection. Biosensors \& Bioelectronics 1997, 12(6), 531-538.

11. Rathgeb, F.; Gauglitz, G. Dyeless optical detection of ammonia in the gas phase using pHresponsive polymers with reflectometric interference spectroscopy. Analytica Chimica Acta 1998, 372, 333-340.

12. Yu, F.; Yao, D.F.; Qian, W.P.; et al. Reflectometry. interference spectroscopy in detection of hepatitis B surface antigen. Clin. Chem. 2000, 46(9), 1489-1490.

13. Lü, X.Y.; Huang, H.F.; Chen, D.M.; et al. Real time in situ kinetic analysis of proteins adsorbed onto the surface of hydroxyapatite film using RIfS. Proceedings of the first international conference on biomaterials (China) July 24-26,2001, Beijing, China.

14. Sharma, C.P.; Sunny, M.C. Albumin adsorption on to aluminum oxide and polyurethane surfaces. Biomaterials 1990, 11, 255-257.

15. Zhang, S.J.; Li, D.J.; Zhao, J.; Gu, H.Q. A study of blood protein adsorption on diamond-like carbon. Chinese Conference on Biomaterials. Panyu. China 1999 Nov; 5-8.

16. Babensee, J.E.; Cornelius, R.M.; Brash, J.L.; Sefton, M.V. Immunoblot analysis of proteins associated with HEMA-MMA microcapsules: Human serum proteins in vitro and rat proteins following implantation. Biomaterials 1998, 19, 839-849. 
17. Kandori, K.; Fujiwara, A.; Mukai, M.; Yasukawa, A.; Ishikawa, T. Evaluation of the adsorption affinity of proteins to calcium hydroxyapatites by desorption and pre-adsorption methods. Colloids and Surfaces B: Biointerfaces 1998, 11, 313-320.

18. Klomp, A.J.A.; Engbers, G.H.M.; Mol, J.; Terlingen, J.G.A.; Feijen, J. Adsorption of proteins from plasma at polyester non-wovens. Biomaterials 1999, 20, 1203-1211.

19. Chittur, K.K. FTIR/ATR for protein adsorption to biomaterial surfaces. Biomaterials 1998, 19, 357-369.

20. Zeng, H.; Chittur, K.K.; Lacefield, W.R. Analysis of bovine serum albumin adsorption on calcium phosphate and titanium surfaces. Biomaterials 1999, 20, 377-384.

21. Kingshott, P.; Heather, A.W.; John, S.T.; Griesser, H.J. Direct detection of proteins adsorbed on synthetic materials by matrix-assisted laser desorption ionization-mass spectrometry. Analytical Biochemistry 1999, 273, 156-162.

22. Oleschuk, R.D.; McComb, M.E.; Chow, A.; Ens, W.; Standing, K.G.; Perresult, H.; Mariois, Y.; King, M. Characterization of plasma proteins adsorbed onto biomaterials by MALDI-TOFMS. Biomaterials 2000, 21, 1701-1710.

23. Ortega-Vinuesa, J.L.; Tengvall, P.; Wälivaara, B.; Lundström, I. Stagnant versus dynamic conditions: a comparative adsorption study of blood proteins. Biomaterials 1998, 19, 251-262.

24. Zhang, M.; Desai, T.; Ferrari, M. Proteins and cells on PEG immobilized silicon surfaces. Biomaterials 1998, 19, 953-960.

25. Elwing, H. Protein absorption and ellipsometry in biomaterial research. Biomaterials 1998, 19, 397-406.

26. Tengyall, P.; Lundström, I.; Liedberg, B. Protein adsorption studies on model organic surfaces: an ellipsometric and infrared spectroscopic approach. Biomaterials 1998, 19, 407-422.

27. Hirano, S.; Zhang, M.; Nakagawa, M.; Miyata, T. Wet spun chitosan-collagen fibers, their chemical $N$-modifications, and blood compatibility. Biomaterials 2000, 21, 997-1003.

28. Gu, H.Q.; Xu, G.F. Biomedical Materials. Publishing Company. Of Translate for Sci and Tech. Tianjing, China. 1993, p316-324.

29. Zhang, J.X.; Tang, J.; Xu, B. Biocompatibility and safety evaluation of chitosan rod. J Biomed Eng. (Chinese) 1996, 13(4), 293-297.

30. Jiang, X.S.; Wang, B.S.; Chen, C.; Li, X.G.; Sun, F.Y. The bioactivity and medical appliance of chitin and its ramification. J Biomed Eng. (Chinese) 1996, 13(4), 353-356.

31. Andrew, C.A.W.; Khor, E.; Hastings, G.W. The influence of anionic chitin derivatives on calcium phosphate crystallization. Biomaterials 1998, 19, 1309-1316.

32. Leroux, L.; Freche, Z., Frèche, M.; Lacout, J.L. Effects of various adjuvants (lactic acid, glycerol, and chitosan) on the injectability of a calcium phosphate cement. Bone 1999 Supplement August; 25(2), 318-348.

33. Chenite, A.; Chaput, C.; Wang, D.; Combes, C.; Buschmann, M.D.; Hoemann, C.D.; Leroux, J.C.; Atkinson, B.L.; Binette, F.; Selmani, A. Novel injectable neutral solutions of chitosan form biodegradable gels in situ. Biomaterials 2000, 21, 2155-2161. 
34. Campos, A.M.D.; Sánchez, A.; Alonso, M.J. Chitosan nanoparticles: a new vehicle for the improvement of the delivery of drugs to the ocular surface. Application to cyclosporin A. International Journal of Pharmaceutics 2001, 224, 159-168.

35. Van der Lubben, I.M.; Verhoef, J.C.; Van Aelst, A.C.; Borchard, G.; Junginger, H.E. Chitosan microparticles for oral vaccination: preparation, characterization and preliminary in vivo uptake studies in murine Peyer's patches. Biomaterials 2001, 22(7), 687-694.

36. Yang, J.; Tian, F.; Chen, S.Q. The mechanism of chitosan hemostasis and its application. Alien Medical: Biomedical and engineering 2001, 24(2), 77-80.

37. Amiji, M.M. Platelet adhension and activation on an amphoteric chitosan derivative bearing sulfonate groups. Colloids and Surfaces B:Biointerfaces 1998, 10, 263-271.

38. Lin, C.W.; Lin, J.C. Surface characterization and platelet compatibility evalution of surfacesulfonated chitosan membrane. J Biomater Sci Polym Ed. 2001, 12(5), 543-557.

39. Green, R.J.; Davies, J.; Davies, M.C.; Robert, C.J.; Tendler, S.J.B. Surface plasmon resonance for real time in situ analysis of protein adsorption to polymer surfaces. Biomaterials 1997, 18, 405413.

40. $\mathrm{Yu}, \mathrm{F}$. The establishment of reflectometry interference spectroscopy and its use in the token of interface biomolecule. M.S.Thesis: Biomedical and Engineering Department of Southeast University, Nanjing, China., July 2000.

41. Huang, S.L.; Chao, M.S.; Ruan, R.C.; Lai, J.Y. Microphase separated structure and protein adsorption of polyurethanes with butadiene soft segment. European Polymer Journal 2000, 36, 285-294.

42. Qian, W.P.; Yao, D.F.; Yu, F.; Xu, B.; Zhou, R.; Bao, X.; Lu, Z.H. Immobilization of antibodies on ultraflat polystyrene surfaces. AACC's Oak Ridge Conference, Bosten, USA. 2000 May; 5-6.

Sample Availability: Available from the author.

(C) 2001 by MDPI (http://www.mdpi.net). Reproduction is permitted for noncommercial purposes. 